Arleana do Bom Parto Ferreira de ALMEIDA ${ }^{1}$ Valéria Régia Franco SOUSA

Luciana DALCIN

Christiano Henrique da Silva JUSTINO

\section{Correspondência para:} VALÉRIARÉGIAFRANCOSOUSA Rua 10, Casa 53 - Bairro Boa Esperança 78 068-727 - Cuiabá - MT regia@ufmt.br

Recebido para publicação: 06/02/2006 Aprovado para publicação: 07/02/2007

\title{
Contaminação por fezes caninas das praças públicas de Cuiabá, Mato Grosso
}

\author{
1 - Universidade Federal de Mato Grosso, Cuiabá - MT
}

\section{Resumo}

\author{
Palavras-chave: \\ Ancylostoma sp. \\ Toxocara sp. \\ Praças públicas.
} O presente trabalho teve o objetivo de avaliar a contaminação das praças públicas de Cuiabá, Estado de Mato Grosso, por fezes de cães. Foram pesquisadas 55 praças, de agosto a novembro de 2006. Destas, 14 localizavam-se no centro da cidade e 41 em diversos bairros periféricos do município. As amostras foram coletadas pela manhã, armazenadas em sacos plásticos sob refrigeração e processadas no Laboratório de Doenças Parasitárias do Hospital Veterinário da UFMT, através das técnicas de Willis-Mollay e Hoffmann, Pons e Janer, 1934. Das 55 praças, 40 possuíam amostras de fezes de cães. Ao todo foram coletadas 121 amostras e em 45 foram observados ovos de helmintos. Destas, 38 (84,4\%) apresentaram ovos de Ancylostoma sp; 07 (15,5\%) de Toxocara sp; 09 (20\%) de Trichuris vulpis e 01 (2,2\%) de Platynossomum sp e Cystoisospora sp. Vinte e duas das 121 amostras encontradas foram nas praças centrais e 99 nas de bairros periféricos, entretanto não houve diferença estatística significativa $(p>0,05)$ entre as mesmas. Este estudo revelou alta contaminação de praças públicas da cidade de Cuiabá por ovos de helmintos, incluindo os de importância em saúde pública, o que indica risco de transmissão dessas zoonoses a população.

\section{Introdução}

As praças e parques públicos contaminados por fezes de cães constituem uma importante via de transmissão de zoonoses parasitárias ${ }^{1}$. O crescente número de cães domiciliados, peridomiciliados e errantes e o aumento do acesso destes a locais públicos tem aumentado o risco de contaminação ambiental e infecção, especialmente para as crianças, por zoonoses $^{2}$, como a Larva Migrans Cutânea (LMC) e a Larva Migrans Visceral (LMV) ${ }^{3}$.

A LMC é causada pelo Ancylostoma sp, helminto que parasita cães e gatos e, ocasionalmente os seres humanos ${ }^{1}$, através da penetração da larva infectante ( $3^{\circ}$ estádio larval) pela pele ${ }^{1,4}$. Após penetração, essas larvas fazem migração pelo tecido subcutâneo acarretando reações inflamatórias caracterizadas por prurido intenso e erupção linear e tortuosa da pele, observadas principalmente nos membros inferiores ${ }^{1,2,3,5}$. Essa dermatose tem sido observada em pacientes que tiveram contato com areia de praias, de depósitos peridomiciliares ou de áreas de recreação ${ }^{5}$.

No município de Taciba, São Paulo, foi relatado a ocorrência de três casos de dermatites em crianças de aproximadamente 10 anos de idade, que se caracterizavam por lesões serpiginosas, com áreas de eritema e prurido intenso, principalmente nos membros inferiores, sendo diagnosticado LMC. Nas praças próximas às residências das crianças acometidas foi verificada a ocorrência de larvas de Ancylostoma sp ${ }^{1}$. Em um estudo realizado com fezes coletadas em 74 praças de Campo Grande, Mato Grosso do Sul, $42(56,8 \%)$ apresentavam contaminação por ovos de Ancylostoma sp e $8(10,8 \%)$ por ovos de Toxocara sp.

A LMV é resultado da migração errática do estágio larval ( $3^{\circ}$ estádio larval), do Toxocara canis ${ }^{6}$. A infecção ocorre pela ingestão de ovos larvados de T. canis presentes no solo, sendo mais frequente em crianças com idade entre um e cinco anos de idade que possuem o hábito de ingerir 
solo contaminado com ovos embrionados de Toxocara sp ${ }^{7}$. Essas larvas eclodirão no intestino e migrarão pela via linfática ou circulação portal para diversos órgãos, principalmente fígado e pulmão e ocasionalmente, coração e sistema nervoso central, dando origem a Síndrome Larva Migrans Visceral, ou afetando o globo ocular e gerando a Síndrome Larva Migrans Ocular (LMO $)^{6,7}$. Sendo já relatado a ocorrência de LMO por Ancylostoma caninum no $\mathrm{Brasil}^{8}$ e a ocorrência de LMV em um paciente adulto que apresentava apenas lesões cutâneas e eosinofilia como elementos clínicos auxiliares no diagnóstico, sendo esta uma apresentação clínica atípica?.

Várias pesquisas têm sido feitas para avaliar a contaminação de parques e praças públicas por ovos de helmintos, principalmente Toxocara sp e Ancylostoma sp. Em Sorocaba, São Paulo, das 30 praças examinadas, 16 apresentavam contaminação por ovos de Toxocara spp. ${ }^{6}$, em Botucatu, 17,5\%7. No Balneário Cassino, Rio Grande do Sul, das 237 amostras coletadas, $86,1 \%$ possuiam ovos e/ou larvas de helmintos, sendo que
$71,3 \%$ apresentavam ovos e larvas de Ancylostoma spp, 43,5\% ovos de Ancylostoma spp, 9,7\% Trichuris spp e 3\% Toxocara spp $^{2}$.

Este trabalho teve por objetivo investigar a ocorrência de contaminação das praças públicas de Cuiabá, Mato Grosso, por ovos de Toxocara spe Ancylostoma sp, agentes causadores da LMV e LMC.

\section{Material e Método}

No presente estudo foram pesquisadas 55 praças do município de Cuiabá, Mato Grosso. Destas, 14 localizavam-se no centro da cidade e $41 \mathrm{em}$ diversos bairros periféricos.

As visitas às praças ocorreram de agosto a novembro de 2005 , sempre no período da manhã. As amostras de fezes caninas encontradas foram armazenadas em sacos plásticos e mantidas sob refrigeração até o momento do processamento no Laboratório de Doenças Parasitárias do Hospital Veterinário da UFMT. Para cada praça foi estipulado um número máximo de seis amostras.

Para a pesquisa de ovos e oocistos

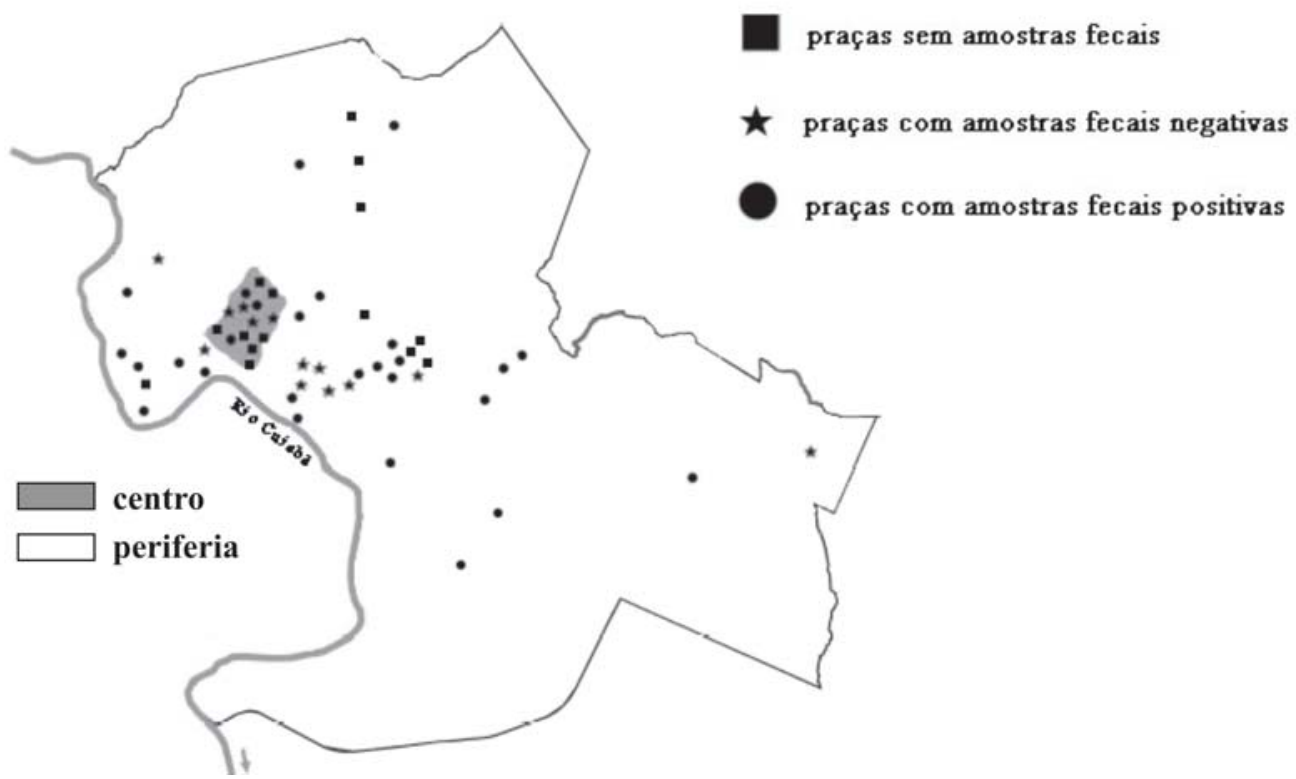

Figura 1 - Distribuição geográfica das praças públicas de Cuiabá analisadas quanto à presença de ovos de helmintos em fezes caninas no período de agosto a novembro de 2006 
foram realizadas as técnicas de Willis-Mollay e Hoffmann, Pons e Janer, 1934, sendo confeccionadas uma lâmina de cada amostra. As lâminas obtidas foram examinadas em microscopia óptica para a presença de ovos de helmintos e oocistos de protozoários.

Os resultados obtidos foram analisados estatisticamente pelo método não paramétrico do qui-quadrado, através do programa Epi info versão 3.3.2.

\section{Resultados e Discussão}

Das 55 praças visitadas, 40 possuíam amostras de fezes caninas, sendo 33 localizadas na periferia e sete no centro da cidade. Ao todo foram coletadas 121 amostras fecais. Em 75 não foram verificados ovos de helmintos nas duas técnicas realizadas, e em 45 amostras observou-se em uma ou ambas as técnicas ovos de helmintos (Figura 1).

Das 45 amostras positivas, $38(84,4 \%)$ apresentaram ovos de Ancylostoma sp; 07 (15,5\%) de Toxocara sp; 09 (20\%) de Trichuris vulpis e 01 (2,2\%) de Platynossomum sp e Cystoisospora sp. Houve co-infecção entre Ancylostoma sp e Trichuris vulpis em $08(17,7 \%)$ e Ancylostoma sp e Toxocara sp em 03 (6,6\%) as amostras.

A alta percentagem de ovos de Ancylostoma sp e Toxocara sp obtida neste trabalho é similar a obtida por Araújo et al. ${ }^{3}$ e Castro, Santos e Monteiro ${ }^{10}$ que analisaram a ocorrência destes parasitos em fezes de cães em praças públicas de Campo Grande, Mato Grosso do Sul e Praia Grande, São Paulo, respectivamente. Blazius et al. ${ }^{2}$ encontraram uma percentagem de $70,9 \%$ e $14,5 \%$ para ovos dos mesmos agentes em amostras de fezes de cães errantes na cidade de Itapema, Santa Catarina.

Além dos estudos com amostras fecais de cães e obtidas de praças públicas para a ocorrência de ovos de Ancylostoma sp e Toxocara sp, têm-se estudado a ocorrência destes helmintos em amostras de solo de praças e áreas de recreação, como demonstrado por Guimarães et al. ${ }^{12}$ que encontraram contaminação por esses agentes em 69,6\% das amostras de solo analisadas. Santarém, Sartor e Bergamo ${ }^{7}$ e Capuano e Rocha ${ }^{13}$ obtiveram uma percentagem de $17,5 \%$ e $16 \%$ das amostras analisadas para a ocorrência de ovos de Toxocara sp em praças de Botucatu e Ribeirão Preto, São Paulo, respectivamente.

O encontro de um elevado número de amostras positivas para ovos de Trichuris vulpis é similar ao de Scaini et al. ${ }^{2}$ que encontraram ovos deste parasito em 23,5\% das amostras fecais em Balneário Cassino, Rio Grande do Sul e Blazius et al. ${ }^{11}$ analisando amostras de fezes de cães errantes de Itapema, Santa Catarina onde obteve $13,9 \%$ de positividade para ovos deste parasito em seu estudo. Esse helminto além de causar danos aos cães pode eventualmente parasitar o homem ${ }^{14}$.

Quanto à contaminação das praças segundo a sua localização, constatou-se que das 121 amostras fecais, 22 encontravam-se nas praças centrais e destas, 03 foram positivas para ovos de Ancylostoma sp. As 99 amostras restantes encontravam-se nas praças periféricas, sendo 42 positivas para ovos de helmintos. Em 35 (83,3\%) houve a presença de Ancylostoma sp, 07 (16,6\%) Toxocara sp, $09(21,45 \%)$ Trichuris vulpis e 1 (2,38\%) Platynossomum sp e Cystoisospora sp. Todas as co-infecções encontradas foram de amostras encontradas em praças periféricas de Cuiabá.

Esta maior contaminação das praças localizadas na periferia da cidade também foi observada por Coelho et al. ${ }^{6} \mathrm{em}$ Sorocaba, São Paulo que analisaram 15 praças centrais e 15 periféricas deste município, encontrando 16 contaminadas, 09 de bairros periféricos e 07 centrais. Não houve diferença significativa $(\mathrm{p}>0,05)$ entre a contaminação das praças de bairros periféricos em relação às centrais de Cuiabá, apesar do número da primeira ser superior à segunda.

Analisando a presença de ovos de helmintos nas praças dos bairros classificados pela renda per capita (Tabela 01), adotada no Perfil Sócio Econômico de Cuiabá ${ }^{15}$ 
Tabela 01 - Freqüência de helmintos em fezes caninas colhidas de praças públicas dos bairros de Cuiabá classificados de acordo com a renda per capita, 2005

\begin{tabular}{|c|c|c|c|}
\hline Faixa de Renda* & Amostras Positivas & Amostras Negativas & Total de Amostras \\
\hline Baixa $^{A}$ & $00(0,0 \%)$ & $01(0,8 \%)$ & $01(0,8 \%)$ \\
\hline Médio-Baixa ${ }^{\mathrm{B}}$ & $13(10,7 \%)$ & $08(6,6 \%)$ & $21(17,3 \%)$ \\
\hline Média $^{C}$ & $24(19,8 \%)$ & $24(19,80 \%)$ & $48(39,6 \%)$ \\
\hline Média-Alta ${ }^{\mathrm{D}}$ & $08(6,6 \%)$ & $39(32,2 \%)$ & $47(38,8 \%)$ \\
\hline Alta ${ }^{\mathrm{E}}$ & $00(0,0 \%)$ & $04(3,3 \%)$ & $04(3,3 \%)$ \\
\hline
\end{tabular}

Faixa de Renda em salário mínimo: A: < 2,91; B: de 2,91 a 5,65; C: de 5,66 a 11,65; D: de 11,66 a 21,94; E: > 21,94

verificou-se que nas duas faixas extremas de renda, isto é, alta e baixa, o número de amostras foi insuficiente para avaliação estatística. Já entre os bairros da faixa média baixa e média comparados aos da faixa média alta, houve um maior número de amostras negativas na última faixa, estatisticamente significativa $(p<0,05)$, sugerindo que nos bairros de menor renda exista uma maior contaminação da população canina por helmintos que pode ser decorrente de dificuldade de acesso à assistência médico veterinária por questões culturais e econômicas.

Comparando as técnicas utilizadas a de Willis-Mollay apresentou uma positividade em $34(75,5 \%)$ e a de Hofmann, Pons e Janer, 1934 em 37 (82,2\%) amostras positivas. Em ambas houve a visualização de ovos de Ancylostoma sp (31), Trichuris vulpis (07) e Platynossomum sp (01) na mesma proporção. Houve divergência na técnica de Willis-Mollay e Hofmann, Pons e Janer, 1934 quanto à observação de ovos de Toxocara sp e oocistos de Cystoisospora sp, sendo que na primeira técnica verificaram-se ovos em seis amostras e oocistos em uma amostra, enquanto na segunda foram observados ovos de Toxocara sp em apenas quatro amostras não havendo a observação de Cystoisospora sp.

As duas técnicas empregadas são habitualmente associadas na rotina. A de Willis-Mollay por se tratar de uma técnica de flutuação é empregada na recuperação de ovos de cestóides e nematóides, contrária à técnica de Hofmann, Pons e Janer, 1934, técnica de sedimentação, utilizada para a recuperação de ovos de trematóides. Apesar de suas características, não houve diferença significativa entre as duas técnicas quanto à recuperação de ovos de helmintos e oocistos de Cystoisospora sp. nem quando analisadas quanto à espécie recuperada ${ }^{16}$.

\section{Conclusão}

O presente estudo revelou alta contaminação de praças públicas da cidade de Cuiabá por ovos de helmintos, incluindo os de importância em saúde pública, o que indica risco de transmissão dessas zoonoses a população. Com base nesses dados, faz necessária a implementação de medidas preventivas e de controle, como a prevenção da defecação de cães em áreas públicas, controle dos cães errantes, promoção de leis protetoras de áreas públicas, além de tratamento periódico dos cães.

\section{Contamination for faecal samples dogs of the public squares of Cuiabá, Mato Grosso}

\section{Abstract}

The present work had the objective to evaluate the contamination of the public squares of Cuiabá, State of Mato Grosso, for faecal samples of dogs. Fifty five squares had been searched, of August the November
Key-words: Ancylostoma sp.

Toxocara sp. Public squares. Zoonosis. 
of 2006. Of these, 14 beed situated in the center of city and 41 in diverse outlying areas of the city. The samples were collected per the morning, stored in processed plastic bags under refrigeration and in the Laboratory of Parasitic Illnesses of the Hospital Veterinarian of the UFMT, through the techniques of Willis-Mollay and Hoffmann, Pons and Janer, 1934. Of the 55 squares, 40 had faecal samples of dogs. To 121 samples had been all collected and in 45 eggs of helminths had been observed. Of these, $38(84,4 \%)$ had presented eggs of Ancylostoma sp; 07 (15,5\%) of Toxocara sp; 09 (20\%) of Trichuris vulpis ones and $01(2,2 \%)$ of Platynossomum sp and Cystoisospora sp. Twenty and two of the 121 joined samples had been in the squares central offices and 99 in the ones of outlying areas, however did not have difference significant statistics $(p>0,05)$ between the same ones. This study it disclosed high contamination of public squares of the city of Cuiabá for eggs of helminths, including of importance in public health, what it indicates risk of transmission of these zoonosis the population.

\section{Referências}

1 SANTARÉM, V. A.; GIUFFRIDA, R.; ZANIN, G. A. Larva migrans cutânea: ocorrência de casos humanos e identificação de larvas de Ancylostoma spp em parque público do município de Taciba, São Paulo. Revista da Sociedade Brasileira de Medicina Tropical, v. 37, n. 2, p. 179-181, 2004

2 SCAINI, C. J. et al. Contaminação ambiental por ovos e larvas de helmintos em fezes de cães na área central do Balneário Cassino, Rio Grande do Sul. Revista da Sociedade Brasileira de Medicina Tropical, v. 36, n. 5, p. 617-619, 2003.

3 ARAÚJO, F. R. et al. Contaminação de praças públicas de Campo Grande, Mato Grosso do Sul, Brasil, por ovos de Toxocara e Ancylostoma em fezes de cães. Revista da Sociedade Brasileira de Medicina Tropical, v. 32, n. 5, p. 581-583, 1999.

4 REY, L. Um século de experiência no controle da ancilostomíase. Revista da Sociedade Brasileira de Medicina Tropical, v. 34, n. 1, p. 61-67, 2001.

5 NUNES, C. M. et al. Ocorrência de larva migrans na areia de áreas de lazer das escolas municipais de ensino infantil, Araçatuba, SP, Brasil. Revista de Saúde Pública, v. 34, n. 6, p.656-658, 2000.

6 COELHO, L. M. P. S. et al. Toxocara spp. eggs in public squares of Sorocaba, São Paulo State, Brazil. Revista do Instituto de Medicina Tropical de São Paulo, v. 43, n. 4, p. 189-191, 2001.

7 SANTARÉM, V. A.; SARTOR, I. F.; BERGAMO, F. M. M. Contaminação, por ovos de Toxocara spp, de parques e praças públicas de Botucatu, São Paulo, Brasil. Revista da Sociedade Brasileira de Medicina Tropical, v. 31, n. 6, p. 529-532, 1998.

8 CASELLA, A. M. B.; MACHADO, R. A.; TSURO, A. et al. Seria o Ancylostoma caninum um dos agentes da neurorretinite sub-aguda difusa unilateral (D.U.S.N.) no Brasil?. Arquivo Brasileiro de Oftalmologia, v. 64 n. 5, p. 473-476, 2001.

9 MACHADO, A. B.; EL ACHKAR, M. E. Larva migrans visceral: relato de caso. Anais Brasileiros de Dermatologia, v. 78, n. 2, p. 215-219, 2003.

10 CASTRO, J. M. ; SANTOS, S. V.; MONTEIRO, N. A. Contaminação de canteiros da orla marítima do Município de Praia Grande, São Paulo, por ovos de Ancylostoma e Toxocara em fezes de cães. Revista da Sociedade Brasileira de Medicina Tropical, v. 38, n. 2 , p. 199-201, 2005.

11 BLAZIUS, R. D. et al. Ocorrência de protozoários e helmintos em amostras de fezes de cães errantes da Cidade de Itapema, Santa Catarina. Revista da Sociedade Brasileira de Medicina Tropical, v. 38, n. 1, p. 73-74, 2005.

12 GUIMARÃES, A. M. et al. Ovos de Toxocara sp e Larvas de Ancylostoma sp em praça pública de Lavras, MG. Revista de Saúde Pública, v. 39, n. 2, p. 293-5, 2005.

13 CAPUANO, D. M.; ROCHA, G. M. Environmental contamination by Toxocara sp. Eggs in Ribeirão Preto São Paulo State, Brazil. Revista do Instituto de Medicina Tropical de São Paulo, v. 47, n. 4, p. 223-226, 2005.

14 DUNN, J. J. et al. Trichuris vulpis from a patient with chronic diarrhea and five dogs. Journal of Clinical Microbiology, v. 40, n. 7, p. 2703-2704, 2002.

15 PREFEITURA MUNICIPAL DE CUIABÁ. Perfil Socioeconômico de Cuiabá. Cuiabá: Instituto de Pesquisa e Desenvolvimento Urbano, 2004. v. 2, 407 p.

16 URQUHART, G. M. et al. Parasitologia Veterinária. 2. ed. Rio de Janeiro: Guanabara Koogan, 1998. p. 239-247. 